\author{
Marleen E. Graat \\ Anke Kröner \\ Peter E. Spronk \\ Johanna C. Korevaar \\ Jaap Stoker \\ Margreeth B. Vroom \\ Marcus J. Schultz
}

\section{Elimination of daily routine chest radiographs in a mixed medical-surgical intensive care unit}

Received: 17 February 2006

Accepted: 11 January 2007

Published online: 28 February 2007

(C) Springer-Verlag 2007

Electronic supplementary material

The online version of this article (doi:10.1007/s00134-007-0542-1) contains supplementary material, which is available to authorized users

M. E. Graat · A. Kröner · M. B. Vroom · M. J. Schultz

University of Amsterdam, Department of Intensive Care Medicine, Academic

Medical Center, Mail stop C3-329,

Meibergdreef 9, 1105 AZ Amsterdam,

The Netherlands

A. Kröner

University of Amsterdam, Deparment of Anesthesiology, Academic Medical Center, Meibergdreef 9, 1105 AZ Amsterdam,

The Netherlands

\section{P. E. Spronk}

Gelre Hospital (Location Lukas), Department of Intensive Care Medicine, Albert Schweitzerlaan 31, 7334 DZ Apeldoorn, The Netherlands

\section{J. C. Korevaar}

University of Amsterdam, Department of Clinical Epidemiology and Biostatistics, Academic Medical Center,

Meibergdreef 9, 1105 AZ Amsterdam, The Netherlands

\section{J. Stoker}

University of Amsterdam, Department of Radiology, Academic Medical Center, Meibergdreef 9, 1105 AZ Amsterdam, The Netherlands

\section{J. Schultz (ه)}

University of Amsterdam, Laboratory of Experimental Intensive Care and Anesthesiology (L.E.I.C.A.), Academic Medical Center,

Meibergdreef 9, 1105 AZ Amsterdam, The Netherlands

e-mail: m.j.schultz@amc.uva.nl Tel.: +31-20-5669111

\footnotetext{
Abstract Objective: To determine the impact of elimination of daily routine chest radiographs (CXRs) in a mixed medical-surgical intensive care unit (ICU) on utility of on demand CXRs, length of stay (LOS) in ICU, readmission rate, and mortality rate. Design and setting: Prospective, nonrandomized, controlled study in a 28-bed ICU. Analysis included data of all admitted ICU patients during 5 months before and after elimination of daily routine CXRs. Results: Before elimination, 2457 daily routine CXRs and 1437 on demand CXRs were obtained from 754 patients. After elimination, 1267 CXRs were
}

obtained from 622 patients. The ratio of CXRs/patient day decreased from $1.1 \pm 0.3$ to $0.6 \pm 0.4(p<0.05)$. Elimination did not result in a change in utility and timing of on demand CXRs. The absolute diagnostic and therapeutic value of on demand CXRs increased with elimination of daily routine CXRs: before intervention, 147 unexpected predefined abnormalities were found $(10.2 \%$ of all on demand CXRs in $15.9 \%$ of all patients), of which 57 (3.9\%) in $6.4 \%$ of all patients led to a change in therapy. After intervention, 156 unexpected predefined abnormalities were found $(11.6 \% ; p<0.05)$, of which $61(4.8 \%)$ in $9.5 \%$ of all patients $(p<0.05)$ led to a change in therapy. The LOS in ICU, readmission rate and ICU, and hospital mortality rate were not influenced by the change in strategy. Conclusions: Elimination of daily routine CXRs reduced the number of CXRs in a mixed medical-surgical ICU, while not affecting readmission rate and ICU and hospital mortality rates.

Keywords Daily routine - On demand - Chest radiograph - ICU . Critical care 


\section{Introduction}

Chest radiographs (CXRs) are frequently obtained as a complement to physical examination of critically ill patients $[1,2]$. There are two different schools of thought regarding the utility of CXRs in the intensive care unit (ICU): The CXRs should be made on indication only, specifically when there is a sound reason to obtain a film (so-called on demand CXRs); or CXRs should be obtained routinely every day, that is, without any specific reason (so-called daily routine CXRs). Argument for the latter strategy is the high prevalence of findings on CXRs of ICU patients [3]; however, interpretation of studies on the usefulness of daily routine CXRs is hampered because of major differences in methodology [4]. Importantly, most studies did not attempt to discriminate between clinically relevant and irrelevant findings. We recently demonstrated that daily routine CXRs hardly ever reveal potentially important abnormalities and seldom result in a change in therapy [5].

While it can be recommended to discontinue a daily routine CXR practice in ICU patients, elimination of these CXRs may have several disadvantages. Firstly, eliminating daily routine CXRs bears the risk that the number of on demand CXRs increases. In addition, elimination of daily routine CXRs might result in on demand CXRs being obtained more frequently during off-time hours, which may cause an inverse rise of costs. Secondly, length of stay (LOS) in ICU, readmission rate and mortality rate might be negatively influenced by this change in CXR practice.

To evaluate the impact of elimination of daily routine CXRs we determined the change in on demand CXR practice in our multidisciplinary ICU, where a daily routine CXR strategy was applied until performance of this study. In addition, we evaluated the diagnostic and therapeutic value of on demand CXRs before and after this intervention. Finally, LOS in ICU, readmission rate, and mortality rate during a daily routine CXR strategy were compared with those during an on demand CXR strategy.

\section{Materials and Methods}

\section{Subjects}

A prospective, nonrandomized, controlled design with an intervention was used for this study. Of all patients, all CXRs taken in the adult ICU department of the Academic Medical Center in Amsterdam, Netherlands, from 1 March 2004 to 31 July 2004 and from 1 September 2004 to 31 January 2005 were studied. This department is a closed-format tertiary care, referral, 28-bed multidisciplinary ICU. The patient population consists of cardiothoracic surgery patients, medical patients (including cardiology patients and pulmonary disease patients), and surgery patients (including trauma patients and neuro- surgery patients). Patients who were admitted during the period in between phases 1 and 2, as well as patients that were readmitted, were not analyzed. The study protocol was approved by the local ethics committee.

\section{Protocol}

The study period was divided into two parts: phase 1, a 5-month phase before the intervention during which the daily routine CXR strategy was practiced; and phase 2 , a 5-month phase which began 1 month after the intervention. The intervention consisted of a change in the ordering practice of CXRs: no standing orders for daily routine CXRs; each (on demand) CXR required a clinical indication, such as admittance to the ICU, insertion of central venous lines, intra-aortic balloon pump or tracheal and chest tubes, an increase in oxygen requirement, or a change in pulmonary secretions with or without fever (see Table E1).

For phases 1 and 2, CXR volume data were collected prospectively. Type of, and reason for, admission was registered for all patients. Severity of illness was scored by means of acute physiology and chronic health evaluation (APACHE) II for all patients. Data on LOS in ICU, readmission to ICU as well as ICU, and hospital mortality rate, were collected from the National Intensive Care Evaluation (NICE) database [6]. The LOS was calculated from day and time of arrival at, and discharge from, ICU. The total number of hours in ICU were divided by 24 to determine the exact LOS in ICU in days.

\section{Diagnostic and therapeutic value of on demand CXR}

Diagnostic and therapeutic value of on demand CXRs was determined as described previously for daily routine CXRs [5]. In short, the attending physician completed a specially developed data sheet on radiological abnormalities which was printed on the back of the normal CXR request form. It was to be ticked whether a certain finding was expected, and whether it was "old" (i.e., already present on preceding CXR) or "new" (i.e., not present on preceding CXR). All CXRs were interpreted by an independent radiologist on the day the on demand CXR was performed. Similar to the ICU physicians, the radiologist structurally interpreted these on demand CXRs for each patient (i.e., the radiologist ticked whether radiological abnormalities were absent or present and, if an abnormality was present, whether it was judged to be an "old" or "new" finding).

If a predefined finding was unexpectedly found, then we determined whether any action was taken because of the new unexpected finding. To do this, two of us (M.G. and M.J.S.) and two independent observers carefully read the medical records, checked the patient data 
management system (Metavision, iMDsoft, Sassenheim, The Netherlands) and searched the hospital information system for the following: orders for sputum cultures or performance of a bronchoalveolar lavage for culture, or start of, or a change in, antimicrobial therapy in case of unexpected infiltrates on the CXR; repositioning of tubes in case of malposition of orotracheal tubes (ignoring planned extubations); ultrasound of the thorax in case of pleural effusion on the CXR, start or change in medication (diuretics); insertion of a pleural drain; and repositioning of devices in the case of malposition of medical devices other than orotracheal tubes (ignoring planned changes such as removal of intravenous lines). The observers were not involved in the daily care of the patients, and ICU physicians were not aware of this part of the observation. As a consequence, the clinical relevance of the predefined abnormalities could not be evaluated in some cases, specifically in cases of large atelectasis and severe pulmonary congestion, since start of physiotherapy, changes in levels of positive end-expiratory pressure, and the use of diuretics might have been triggered by other (clinical) findings.

\section{Statistical analysis}

All data are expressed as means $( \pm \mathrm{SD})$, or medians (interquartile ranges). A Mann-Whitney U-test was used for analyzing continuous variables. A chi-square test was used to compare the groups in phase 1 and phase 2 . The incidences of expected and unexpected findings, and clinically important abnormalities, were compared by chi-square test. A $p$-value $<0.05$ was considered to be statistically significant. All calculations were performed using SPSS version 12.0.1 software (SPSS, Chicago, Ill.).

\section{Results}

Study population

We evaluated 1376 patients over the two periods. Patient profiles on entering this study are summarized in Table 1. A total of 3894 CXRs were obtained from 754 patients in phase 1; these included 2457 daily routine CXRs and 1437 on demand CXRs. A total of 1267 CXRs were obtained from 622 patients in phase 2 . These CXRs were, by definition, all on demand CXRs.

\section{Utility of CXRs}

The number of CXRs per day for the whole ICU declined from $22.6 \pm 4.9$ to $8.2 \pm 3.2$ ( $p<0.05$; Fig. 1$)$. Adjusting for patient volume, the ratio of CXRs per patient day decreased from $1.1 \pm 0.3$ to $0.6 \pm 0.4$ after the intervention $(p<0.05)$. The median number of CXRs per patient for the complete stay in ICU declined from 3 (range 2-5) during phase 1 , to 1 (range 1-2) after the intervention. The number of on demand CXRs increased minimally after the intervention, and the distribution over $24 \mathrm{~h}$, did not change (see ESM, Fig. E1).

Table 1 Demographic data. APACHE-II Acute Physiology and Chronic Health Evaluation II, CXRs chest radiographs, CI confidence interval

\begin{tabular}{lccc}
\hline & Phase 1 & Phase 2 & Significance $(p)$ \\
\hline No. of patients & 754 & 622 & \\
Age (years; mean, SD) & $60(16)$ & $62(16)$ & 0.02 \\
Gender (male; $n$ ) & $475(63 \%)$ & $398(64 \%)$ & 0.70 \\
CXRs while patients being mechanically ventilated $(n)^{\mathrm{a}}$ & $3194(82 \%)$ & $1115(88 \%)$ & $<0.001$ \\
APACHE-II score & $16.4 \pm 6.9$ & $16.4 \pm 7.2$ & 1.00 \\
Patient subgroups & $317(42 \%)$ & $306(49 \%)$ & 0.01 \\
$\quad$ Cardiac surgery $(n)$ & $197(26 \%)$ & $119(19 \%)$ & \\
Medical $(n)$ & $144(19 \%)$ & $131(21 \%)$ & \\
Surgical $(n)$ & $69(9 \%)$ & $46(7 \%)$ & \\
Neurosurgical/neurology $(n)$ & $27(4 \%)$ & $20(3 \%)$ & \\
Other $(n)$ & $1.9(1.0-4.6)$ & $1.9(0.9-4.6)$ & 0.95 \\
Length of stay in ICU (days; median IQR) & $94(12 \%)$ & $62(10 \%)$ & 0.49 \\
Mortality & $132(18 \%)$ & $104(17 \%)$ & 0.70 \\
ICU $(n)$ & $181(24 \%)$ & $155(25 \%)$ & 0.69 \\
Hospital $(n)$ & $0.73(0.59-0.90)$ & $0.67(0.53-0.83)$ & \\
Predicted hospital mortality $(\%)$ & & \\
Observed/predicted ratio $(95 \% \mathrm{CI})$ & & \\
\hline
\end{tabular}

${ }^{a}$ All patients were mechanically ventilated at any time during stay in ICU. Expressed is the percentage of CXRs during which patients were on the ventilator while the CXR was performed 


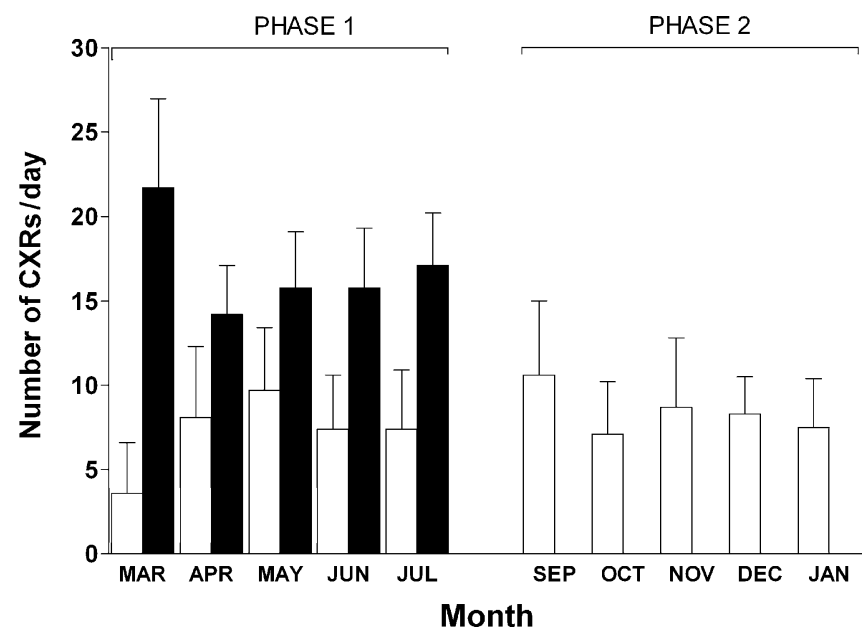

Fig. 1 Number of CXRs/day during the study. Phase 1: daily routine CXR strategy, i.e., a daily routine CXR was made every morning, from March to July; phase 2: on demand CXR strategy, i.e. each CXR required a clinical indication, from September to January. Open bars: mean number ( \pm SD) of on demand CXRs/day; closed symbols: mean number $( \pm \mathrm{SD})$ of all CXRs/day

Diagnostic and therapeutic value of on demand CXRs

The diagnostic and therapeutic value of on demand CXRs increased with elimination of daily routine CXRs (Tables 2, 3). Before intervention, 38 expected predefined abnormalities were found $(2.6 \%$ of all on demand CXRs in $4.9 \%$ of all patients), and after the intervention 64 expected predefined abnormalities were found (5.0\%; $p<0.05)$ in $9.5 \%$ of cases $(p<0.05)$. All these findings led to a change in therapy. Before intervention, 147 unexpected predefined abnormalities were found $(10.2 \%$
Table 3 Unexpected findings on on demand chest radiographs resulting in a chance in therapy. $N D$ not defined

\begin{tabular}{|c|c|c|}
\hline Abnormalities & $\begin{array}{l}\text { Phase } 1 \\
(n=1437) \\
\text { Resulting in a } \\
\text { change in therapy }\end{array}$ & $\begin{array}{l}\text { Phase } 2 \\
(n=1267) \\
\text { Resulting in a } \\
\text { change in therapy }\end{array}$ \\
\hline Large atelectasis & ND & ND \\
\hline Large infiltrates & $10(0.7 \%)$ & $14(1.1 \%)$ \\
\hline Pulmonary congestion & ND & ND \\
\hline Pleural effusion & $11(0.8 \%)$ & $12(0.9 \%)$ \\
\hline $\begin{array}{l}\text { Pneumothorax or } \\
\text { pneumomediastinum }\end{array}$ & $11(0.8 \%)$ & $9(0.7 \%)$ \\
\hline $\begin{array}{l}\text { Malposition of invasive } \\
\text { devices }\end{array}$ & $25(1.7 \%)$ & $29(2.3 \%)$ \\
\hline Total no. of abnormalities & 57 & 64 \\
\hline $\begin{array}{l}\text { Total no. of CXRs with } \\
\text { abnormalities }\end{array}$ & $56(3.9 \%)$ & $61(4.8 \%)$ \\
\hline $\begin{array}{l}\text { Total no. of patients with } \\
\text { CXRs with abnormalities }\end{array}$ & $48(6.4 \%)$ & $59(9.5 \%)^{\mathrm{c}}$ \\
\hline
\end{tabular}

${ }^{a}$ Absolute number of chest radiographs (CXRs; percentage of all daily routine CXRs)

${ }^{\mathrm{b}}$ Absolute number of patients percentage of all patients with ondemand CXRs)

${ }^{\mathrm{c}} p<0.05$ vs phase 1

of all on demand CXRs in $15.9 \%$ of all patients), of which 57 (4.0 in 6.4\%) led to a change in therapy. After intervention 156 unexpected predefined abnormalities were found $(11.6 \%$ of all on demand CXRs in $19.1 \%$ of all patients), of which 64 (4.8 in 9.5\%; $p<0.05)$ led to a change in therapy. Subgroup analysis revealed no differences between phases 1 and 2, except for medical patients, in which there was a significant rise in the number of on demand CXRs that showed an unexpected

Table 2 Expected and unexpected findings on on demand chest radiographs

\begin{tabular}{|c|c|c|c|c|c|c|}
\hline \multirow[b]{2}{*}{ Abnormalities } & \multirow[b]{2}{*}{ Expected } & \multicolumn{3}{|c|}{ Phase $1(n=1437)$} & \multicolumn{2}{|c|}{ Phase $2(n=1267)$} \\
\hline & & $\begin{array}{l}\text { Expected+ } \\
\text { found }\end{array}$ & $\begin{array}{l}\text { Unexpected+ } \\
\text { found }\end{array}$ & Expected & $\begin{array}{l}\text { Expected+ } \\
\text { found }\end{array}$ & $\begin{array}{l}\text { Unexpected+ } \\
\text { found }\end{array}$ \\
\hline Large atelectasis & $37(2.6)$ & $2(0.1)$ & $13(0.9)$ & $49(3.9)$ & $3(0.2)$ & $15(1.2)$ \\
\hline Large infiltrates & $57(4.0)$ & $3(0.2)$ & $21(1.5)$ & $69(5.4)$ & $5(0.4)$ & $27(2.1)$ \\
\hline Pulmonary congestion & $98(6.8)$ & $8(0.6)$ & $25(1.7)$ & $104(8.2)$ & $14(1.1)$ & $22(1.7)$ \\
\hline Pleural effusion & $41(2.9)$ & $3(0.2)$ & $17(1.2)$ & $43(3.4)$ & $4(0.3)$ & $27(2.1)$ \\
\hline $\begin{array}{l}\text { Pneumothorax or } \\
\text { pneumomediastinum }\end{array}$ & $68(4.7)$ & $4(0.3)$ & $17(1.2)$ & $39(3.1)^{\mathrm{c}}$ & $3(0.2)$ & $12(0.9)$ \\
\hline Malposition of invasive devices & $350(24.4)$ & $18(1.3)$ & $54(3.8)$ & $392(30.9)^{\mathrm{c}}$ & $35(2.7)^{\mathrm{c}}$ & $52(4.1)$ \\
\hline Total no. of abnormalities & 651 & 38 & 147 & 696 & $64^{c}$ & 155 \\
\hline $\begin{array}{l}\text { Total no. of CXRs with } \\
\text { abnormalities }\end{array}$ & $641(44.6)$ & $38(2.6)$ & $133(9.2)$ & $384(30.3)^{\mathrm{c}}$ & $63(5.0)^{\mathrm{c}}$ & $147(11.6)^{\mathrm{c}}$ \\
\hline $\begin{array}{l}\text { Total no. of patients with CXRs } \\
\text { with abnormalities }\end{array}$ & $580(76.9)$ & 37 (4.9) & $120(15.9)$ & $223(35.9)^{\mathrm{c}}$ & $58(9.5)^{\mathrm{c}}$ & $119(19.1)$ \\
\hline
\end{tabular}

Numbers in parentheses are percentages

a Absolute number of chest radiographs (CXRs; percentage of all daily routine CXRs)

${ }^{b}$ Absolute number of patients (percentage of all patients with on demand CXRs)

${ }^{c} p<0.05$ vs phase 1 
predefined major abnormality ( $p<0.05$ vs phase 1 ; see ESM, Table E2).

\section{LOS in ICU, readmission rate and mortality rate}

The LOS in ICU was not different in phase 1 as compared with phase 2 (Table 1). Total readmission rate was similar (8.4\% in phase 1 vs $7.6 \%$ and phase 2 , risk difference $0.8 \%$ (95\% CI: $2.1-3.7 \%, P=0.6$ ), and did not change with the intervention for the different subgroups. There were no statistically significant differences in ICU and hospital mortality rates before and after the intervention (Table 1).

\section{Discussion}

The present study demonstrates the impact of elimination of daily routine CXRs in a mixed medical-surgical ICU. We found a sharp decline in the total number of CXRs, while only a minimal increase in the number of on demand CXRs was observed. In addition, the number of CXRs in off-hours was similar between the two periods. Elimination of daily routine CXRs did neither affect LOS in ICU and readmission rate nor ICU and hospital mortality rate.

Although the diagnostic and therapeutic value of on demand CXRs was significantly higher after the intervention, we considered this difference clinically irrelevant. When one considers the increase in diagnostic and therapeutic value of on demand CXRs after elimination of daily routine CXRs indirect proof of the "value" of daily routine CXRs, one must also recognize its futility regarding the therapeutic value. Indeed, the percentage of CXRs with unexpected findings that truly led to a change in therapy was similar in the two study phases. Since readmission rate and mortality rate remained unchanged after the intervention, we conclude that the true value of daily routine CXRs in our multidisciplinary ICU is very low. Interestingly, only in medical patients did the number of CXRs that showed an unexpected predefined major abnormality increase after elimination of daily routine CXRs. The reason for this finding remains unexplained. The distribution of abnormalities encountered on CXRs of these patients was similar in the two study phases; however, neither readmission rate nor differences in raw or risk-adjusted ICU and hospital mortality rates of medical patients was affected by the change in CXR practice.

One interesting finding was the decrease in abnormalities presumed to be present on CXRs. Indeed, a 30\% reduction in expected predefined findings was observed in phase 2 . This finding remains unexplained and we can only speculate on its cause. Firstly, it may be that physicians learned from experience that many of their expectations proved to be untrue during the actual carrying out of the study. This may have caused them to be more reluctant in scoring for expected findings. Alternatively, physicians may have become less enthusiastic about the study, which might have resulted in failure to comply with study rules at some moments (i.e., they did not fill in the back of the formal CXR request form); however, there was no change in expectations of physicians regarding abnormalities that truly led to a change in therapy. More importantly, if the backside of the formal forms were not filled out, as a rule the CXR was simply not obtained. Indeed, collection of data was complete regarding this issue, there were no on demand CXRs without a completed form.

Our study has, at least partially, overlap with two other studies $[7,8]$. Price et al. performed a nonrandomized controlled study on the financial impact of elimination of daily routine CXRs [7]. They showed that elimination of daily routine CXRs in a pediatric ICU resulted in decreased variability in ordering practice, fewer CXRs per patient, and an accompanying cost savings, while not influencing LOS. In addition, cost reduction with the change in radiology policy was significant in their study. This is in line with our results, since we found a substantial decline in radiology costs (see ESM). Besides the fact that this study was performed in a pediatric ICU, making generalization of study results difficult, their study did not include all patient categories. Indeed, postoperative cardiovascular surgery patients continued to receive daily routine CXR. We specifically included this patient group in our study because cardiovascular patients form one of the largest categories in many adult ICU. Krivopal et al. performed a randomized controlled trial to determine whether there is any difference in diagnostic, therapeutic, and outcome efficacy between protocols utilizing daily routine CXRs and those utilizing on demand CXRs in mechanically ventilated patients [8]. In their study a daily routine CXR strategy compared with an on demand CXR strategy was not associated with a negative effect on LOS or mortality; however, this study was small, including not more than 94 patients.

We did not collect information on less evident findings on CXRs. Less evident findings (such as atelectasis less than two lobes, infiltrates less than one lobe, or small pleural effusions [5]), however, might still influence daily management of ICU patients. Since LOS in ICU was not altered for the whole group, readmission rate and mortality rate remained unchanged after the intervention, we suggest that changes of less evident CXR findings are not at all important, at least in our ICU. In other ICUs, such as open-format ICUs, less evident findings might be of more clinical importance, however; therefore, our results must be interpreted with caution, it might be that our results are not easily translated to other types of ICU.

Several important drawbacks of our study must be mentioned. Firstly, our study did not include a strict method for tracking complications as a result of elimination of daily routine CXRs. Indeed, several abnormalities might have been missed (or discovered too late) which might (or do) have impact on clinical outcome. Examples of these types of abnormalities include pneumothorax 
causing weaning problems, the malposition of devices such as central venous lines, causing extravasation of fluid, or orotracheal tubes, potentially causing injury to the vocal cords. Considering these examples, such a strict method may mandate a daily check of all invasive devices. Although possible complications of elimination of daily routine CXRs could be discussed in daily bedside rounds, daily radiology conferences and daily multidisciplinary meetings during the performance of our study, no clinically important complications were reported as the result of elimination of daily routine CXRs; thus, although we assume that the elimination of daily routine CXRs does not cause any complications, we cannot be certain that this was truly the case. Secondly, as mentioned previously, it is of importance to realize that results that come from one center may simply not be similar for other centers: differences in staffing; especially during off-hours, and differences in case mix may be of great influence on outcome when abandoning daily routine CXRs. Thirdly, as mentioned previously, we found a reduction in expected predefined finding in phase 2 . We assumed that the cause of this reduction might be that the physicians became less enthusiastic about the study, which might be seen as a limitation of the study.

\section{Conclusion}

In conclusion, in our mixed medical-surgical ICU elimination of daily routine CXRs leads to a sharp decline in the total number of CXRs, while only minimally increasing the number of on demand CXRs. Although we cannot be certain whether we missed important findings by abandoning daily routine CXRs, its elimination did neither affect LOS in ICU, nor readmission rate and ICU and hospital mortality rates.

\section{References}

1. Trotman-Dickenson B (2003) Radiology in the intensive care unit (Part I) J Intensive Care Med 18:198-210

2. Graat ME, Spronk PE, Schultz MJ (2005) Current practice of chest radiography in critically ill patients in the Netherlands: a postal survey. Chest 128:S296

3. Hall JB, White SR, Karrison T (1991) Efficacy of daily routine chest radiographs in intubated, mechanically ventilated patients. Crit Care Med 19:689-693
4. Graat ME, Stoker J, Vroom MB, Schultz MJ (2005) Can we abandon daily routine chest radiography in intensive care patients? J Intensive Care Med 20:238-246

5. Graat M, Wolthuis E, Choi G, Korevaar JC, Spronk PE, Stoker J, Vroom M, 7. Price MB, Grant MJ, Welkie K (1999) Schultz M (2005) The clinical value of daily-routine chest radiographs in a mixed medical-surgical intensive care units is low. Crit Care 10:1-7
6. Arts D, de Keizer N, Scheffer GJ de Jonge E (2002) Quality of data collected for severity of illness scores in the Dutch National Intensive Care Evaluation (NICE) registry. Intensive Care Med 28:656-659

\section{Financial impact of elimination of} routine chest radiographs in a pediatric intensive care unit. Crit Care Med 27:1588-1593

8. Krivopal M, Shlobin OA, Schwartzstein RM (2003) Utility of daily routine portable chest radiographs in mechanically ventilated patients in the medical ICU. Chest 123:1607-1614 